\title{
Comparative effectiveness of glatiramer acetate and interferon beta formulations in relapsing- remitting multiple sclerosis
}

Tomas Kalincik, Vilija Jokubaitis, Guillermo Izquierdo, Pierre Duquette, Marc Girard, Pierre Grammond, Alessandra Lugaresi, Celia Oreja-Guevara, Roberto Bergamaschi, Raymond Hupperts, Francois Grand'Maison, Eugenio Pucci, Vincent Van Pesch, Cavit Boz, Gerardo Iuliano, Ricardo Fernandez-Bolanos, Shlomo Flechter, Daniele Spitaleri, Edgardo Cristiano, Freek Verheul, Jeannette Lechner-Scott, Maria Pia Amato, Jose Antonio Cabrera-Gomez, Maria Laura Saladino, Mark Slee, Fraser Moore, Orla Gray, Mark Paine, Michael Barnett, Eva Havrdova, Dana Horakova, Timothy Spelman, Maria Trojano* and Helmut Butzkueven*

These authors contributed equally to the manuscript. On behalf of the MSBase Study Group $\dagger$

\section{Abstract}

Background: The results of head-to-head comparisons of injectable immunomodulators (interferon $\beta$, glatiramer acetate) have been inconclusive and a comprehensive analysis of their effectiveness is needed. Objective: We aimed to compare, in a real-world setting, relapse and disability outcomes among patients with multiple sclerosis (MS) treated with injectable immunomodulators.

Methods: Pairwise analysis of the international MSBase registry data was conducted using propensityscore matching. The four injectable immunomodulators were compared in six head-to-head analyses of relapse and disability outcomes using paired mixed models or frailty proportional hazards models adjusted for magnetic resonance imaging variables. Sensitivity and power analyses were conducted.

Results: Of the 3326 included patients, 345-1199 patients per therapy were matched (median pairwisecensored follow-up was 3.7 years). Propensity matching eliminated $>95 \%$ of the identified indication bias. Slightly lower relapse incidence was found among patients treated with glatiramer acetate or subcutaneous interferon $\beta$-1a relative to intramuscular interferon $\beta$-1a and interferon $\beta-1 \mathrm{~b}(p \leq 0.001)$. No differences in 12-month confirmed progression of disability were observed.

Conclusion: Small but statistically significant differences in relapse outcomes exist among the injectable immunomodulators. MSBase is sufficiently powered to identify these differences and reflects practice in tertiary MS centres. While the present study controlled indication, selection and attrition bias, centredependent variance in data quality was likely.

Keywords: Multiple sclerosis, treatment outcomes, patient registry, real-world date, propensity score, relapses, disability

Date received: 7th July 2014; revised: 23rd September 2014; accepted: 22nd October 2014

\section{Introduction}

Interferon $\beta$ (IFN $\beta$ ) and glatiramer acetate (GA) are commonly used in the treatment of relapsing-remitting multiple sclerosis (MS). While numerous randomised clinical trials (RCTs) generated seminal evidence about their efficacy relative to placebo, the evidence concerning their head-to-head comparisons is limited. ${ }^{1-6}$ The high cost and assessment intensity of RCTs pose obvious limitations in relation to duration of existing RCTs. This translates into a limited power to detect treatment effect differences which are either of modest size or delayed. An example of such
Multiple Sclerosis Journal $1-13$

DOI: $10.1177 /$ 1352458514559865

(C) The Author(s), 2014. Reprints and permissions: http://www.sagepub.co.uk/ journalsPermissions.nav

Correspondence to:

Tomas Kalincik

Department of Medicine,

University of Melbourne,

Melbourne, Australia, and

Department of Neurology,

Royal Melbourne Hospital,

Melbourne, L4 Centre,

Melbourne Brain Centre at

Royal Melbourne Hospital,

Grattan St, Parkville VIC

3050, Australia.

tomas.kalincik@unimelb. edu.au

Tomas Kalincik

Vilija Jokubaitis

Timothy Spelman

Department of Medicine, University of Melbourne,

Melbourne, Australia and

Department of Neurology,

Royal Melbourne Hospital,

Melbourne, Australia

Guillermo Izquierdo

Hospital Universitario Virgen

Macarena, Sevilla, Spain

Pierre Duquette

Marc Girard

Hôpital Notre Dame,

Montreal, Canada

Pierre Grammond

Hotel-Dieu de Levis, Quebec, Canada

Alessandra Lugaresi MS Center, Neuroscience, Imaging and Clinical Sciences, University 'G. d'Annunzio', Chieti, Italy

Celia Oreja-Guevara

University Hospital San Carlos, IdISSC, Madrid, Spain

Roberto Bergamaschi

National Neurological Institute

C. Mondino, Pavia, Italy

Raymond Hupperts

Orbis Medical Center,

Sittard, the Netherlands

Francois Grand'Maison Neuro Rive-Sud, Hôpital Charles LeMoyne, Quebec, Canada

Eugenio Pucci

Ospedale di Macerata,

Macerata, Italy

Vincent Van Pesch

Cliniques Universitaires

Saint-Luc, Brussels, Belgium

Cavit Boz

Karadeniz Technical 
University, Trabzon, Turkey Gerardo Iuliano Ospedali Riuniti di Salerno, Salerno, Italy

Ricardo Fernandez-Bolanos Hospital Universitario Virgen de Valme, Seville, Spain Shlomo Flechter

Assaf Harofeh Medical Center, Beer-Yaakov, Israel

Daniele Spitaleri AORN San Giuseppe Moscati, Avellino, Italy

Edgardo Cristiano Hospital Italiano, Buenos Aires, Argentina

Freek Verheul

Groen Hart Ziekenhuis, Gouda, the Netherlands

Jeannette Lechner-Scott John Hunter Hospital, Newcastle, Australia

Maria Pia Amato Department NEUROFARBA, Section of Neurosciences, University of Florence,

Florence, Italy

Jose Antonio Cabrera-

Gomez

Centro Internacional de Restauracion Neurologica,

Havana, Cuba

Maria Laura Saladino INEBA, Buenos Aires,

Argentina

Mark Slee

Flinders University and

Medical Centre, Adelaide,

Australia

Fraser Moore

Jewish General Hospital,

Montreal, Canada

Orla Gray

Craigavon Area Hospital, Portadown, UK

\section{Mark Paine}

St Vincent's Hospital,

Melbourne, Australia

Michael Barnett

Brain and Mind Research

Institute, Sydney, Australia

Eva Havrdova

Dana Horakova

Department of Neurology

and Center of Clinical

Neuroscience, 1st Faculty of

Medicine, General University

Hospital and Charles

University in Prague, Czech Republic

Maria Trojano

Department of Basic Medical Sciences, Neuroscience and Sense Organs, University of Bari, Bari, Italy/These authors contributed equally to the manuscript. effects is the difference in relapse and disability outcomes in patients treated with IFN $\beta$ and GA. In particular, the observed differences in relapse activity, as shown in RCTs, are relatively small, and some of the conclusions drawn from RCTs are contradictory. 3,4 The only difference in disability outcomes was found between IFN $\beta$ - 1 b vs. IFN $\beta$-1a IM, as the typical RCT duration of 2 years has potentially limited their power to detect changes in long-term disability accrual. ${ }^{3}$

The MSBase global clinical practice cohort accumulates longitudinal data from diverse populations over long time periods. Analyses based on such registries are well placed to evaluate treatment effectiveness in the context of real-world medication use, long-term follow-up, and a variety of clinical scenarios. ${ }^{7}$ However, valid and unbiased conclusions can only be drawn on the precondition that appropriate procedures aiming at elimination of indication bias have been implemented. To this end, propensity scorebased methods ${ }^{8}$ have previously been used to generate quasi-randomised studies of MS outcomes. ${ }^{9-11} \mathrm{We}$ have recently demonstrated feasibility of propensitybased matching for evaluation of treatment effectiveness in the MSBase dataset, showing that two dosages of IFN $\beta$-1a SC were equivalent in relapse rate and disability outcomes, thus mirroring the outcomes of the pivotal registration study. ${ }^{12,13}$

Here we present a propensity score-matched analysis comparing the effectiveness of IFN $\beta$ and GA preparations in a series of pairwise head-to-head cohort studies conducted in MSBase.

\section{Patients and methods}

The MSBase registry ${ }^{14}$ is registered with WHO International Clinical Trials Registry Platform [ACTRN12605000455662], and was approved by the Melbourne Health Human Research Ethics Committee [2006.044], and by the local ethics committees in all participating centres (or exemptions granted, according to local regulations). If required, written informed consent was obtained from enrolled patients.

\section{Database and study population}

Longitudinal data from 21,938 patients $(122,561$ patientyears) were extracted from the MSBase registry in April $2013 ; 81 \%$ of patients were enrolled after year 2000 and $79 \%$ had their information updated since 2008.

Inclusion criteria reflected the criteria most commonly employed by RCTs and comprised diagnosis of MS or clinically isolated syndrome (using the 2005 or 2010 McDonald criteria ${ }^{15,16}$ ), relapsing MS course, therapy with IFN $\beta$ or GA as a first-ever disease-modifying agent, at least 6-month persistence on the initial therapy, time from initial symptoms to treatment start $<10$ years, at least one relapse recorded during the 2 years preceding the treatment initiation, and availability of the minimal dataset. The minimal dataset consisted of sex, age, date of first MS symptoms, dates of relapses, clinical MS course, disability at baseline quantified with Expanded Disability Status Scale (EDSS), and treating MS centre. Baseline EDSS was recorded within 6 months of treatment initiation. In addition, an on-treatment follow-up visit with EDSS recorded at least 6 months after the treatment initiation was required. ${ }^{17}$

All information was recorded as part of routine practice, using real-time or near real-time data entry in association with clinical visits. The MSBase protocol stipulates minimum annual updates of the minimum dataset, but patients with less frequent updates were not excluded from the analysis. Data entry portal was either the iMed patient record system or the MSBase online data entry system.

A relapse was defined as occurrence of new symptoms or exacerbation of existing symptoms persisting for $>24$ hours, in the absence of concurrent illness or fever, and occurring at least 30 days after a previous relapse. ${ }^{18}$ Disability was scored by accredited scorers using EDSS (online Neurostatus certification was required at each centre), excluding any EDSS score recorded within 30 days of a previous relapse. Progression of EDSS was defined as an increase of $\geq 1$ EDSS step ( $\geq 1.5$ EDSS step if baseline EDSS was 0 ) sustained for $\geq 12$ months. Onset of secondary progressive MS was defined as at least 1 year of disability progression with or without superimposed relapses following previous relapsing-remitting course, ${ }^{17}$ and was assessed by the treating neurologists. Individual annualised relapse rate (ARR) was calculated as the overall number of recorded relapses between the treatment initiation and one of the following: discontinuation of therapy, end of follow-up, or 5 years from treatment initiation (whichever occurred first). The 5-year censoring was applied in order to ameliorate regression to mean, ${ }^{12}$ which would predominantly impact ARR during longsustained treatment. Categorised evaluations of magnetic resonance imaging (MRI) and cerebrospinal fluid were reported by treating neurologists and were used to better characterise the treatment cohorts.

Quality assurance procedures were followed. Only information from centres with at least 10 active records was used, as stipulated in the study protocol. 
A date of onset was required for all recorded events, including relapses, visits, changes in disease course, and changes in treatment. In addition, a series of automated procedures was applied to identify any invalid or inconsistent entries.

\section{Statistical analysis}

Treatment outcomes were analysed in a series of pairwise models comparing populations matched on their propensity of receiving either of the compared treatments. The hypotheses were postulated prior to inspection of the analysed data. The matching procedures were performed using R 3.0.2 ${ }^{19}$ with MatchIt ${ }^{20}$ and Zelig $^{21}$ packages. Propensity scores were calculated using logistic regression models with the outcome variables representing allocation to either of the compared agents and the independent variables being age, sex, disease duration, ARR over two pre-treatment years, categorised EDSS, disease course, and MS centre. The individual propensity scores were calculated as weighted sums of coefficients of the variables with nonzero weights (at $p \leq 0.1$ ). Patients in the compared treatment group pairs were then matched in a variable 1-3:1 ratio using nearest neighbour matching without replacement and discarding from both groups the cases outside the 0.1 caliper (i.e. with no match within 0.1 standard deviation of the propensity score). Closeness of match was evaluated using cumulative and average propensity distances between the groups. ${ }^{22}$ For each of the matched patient pairs, the common on-treatment follow-up period was determined as the shorter of the two individual follow-up periods. After assessing normality of data distribution, disease outcomes were compared between the propensity score-matched treatment groups. ARR (based on all relapses or relapses treated with corticosteroids) were compared using weighted mixed model with clusters for matched pairs. The proportions of patients free from relapses (censored latest at 5 years) or 12-month confirmed disability progression were evaluated with weighted frailty proportional hazards models. Proportionality of hazards was tested with Schoenfeld's global test and where violated, model with Weibull distribution was applied. All analyses were adjusted for the categorised number of $\mathrm{T} 2$ lesions (missing, 1-8, 29) recorded on baseline brain MRI. The hypotheses were tested at the $p \leq 0.05$ two-tailed level of statistical significance, after controlling false discovery rate within the primary analyses with BenjaminiHochberg procedure.

Ad-hoc power estimation was carried out for the analyses of ARR and was expressed as the minimum detectable effect at $\alpha=0.05$ and $1-\beta=0.95$. Sensitivity analysis was carried out in a subpopulation of patients

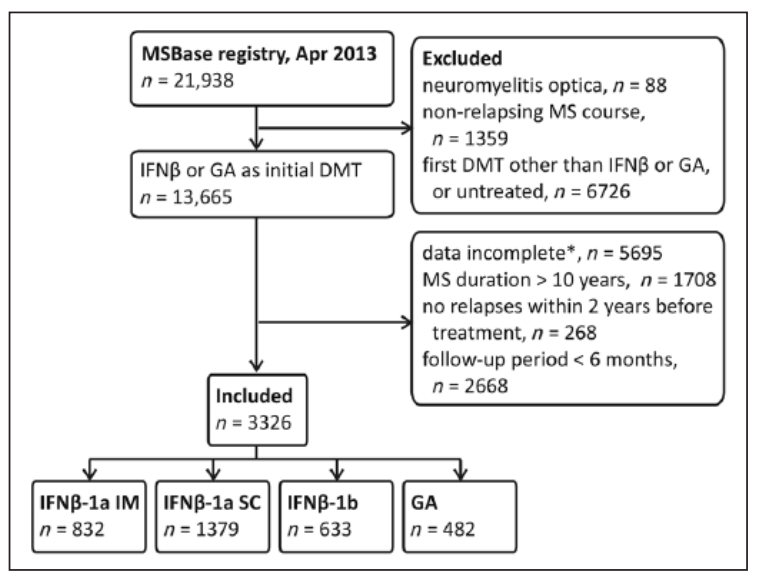

Helmut Butzkueven Department of Medicine, University of Melbourne, Melbourne, Australia, Department of Neurology, Royal Melbourne Hospital, Melbourne, Australia, and Department of Neurology, Box Hill Hospital, Monash University, Box Hill, Australia

†Contributing members of the MSBase Study Group are listed in the Acknowledgements.

Figure 1. CONSORT chart of patient disposition. *The majority of the patients excluded due to incomplete datasets did not have suitable baseline and/or follow-up disability information recorded (for the definition see the inclusion criteria in the Patients and methods section).

DMT: disease-modifying therapy; GA: glatiramer acetate; IFN: interferon; MS: multiple sclerosis; SC: subcutaneous.

matched at a fixed 1:1 ratio. Hodges-Lehmann $\Gamma$ for Rosenbaum bounds was estimated for the ARR analyses to evaluate the robustness of the results in relation to any non-recognised confounders of treatment assignation. $^{23,24}$

\section{Results}

Out of the 21,938 screened patients, 3326 patients (with cumulative follow-up of 15,247 patient-years) from 49 MS centres in 22 countries (online eTable 1) fulfilled the inclusion criteria (for CONSORT diagram see Figure 1). Exclusion of patients was proportional across treatment groups. The median follow-up was 3.7 years (interquartile range 2.26.3), with mean gap between visits of 5.8-7.0 months. As shown in Table 1, the treatment groups differed in several baseline demographic and clinical characteristics. Allocation to therapy (and thus the calculated propensity score) was mostly determined by treating MS centre, followed by disability, pretreatment relapse rate, MS course, and age. For instance, patients treated with IFN $\beta-1 b$ were more frequently those with more severe disability compared with those initiated on other preparations, patients receiving IFN $\beta$-1a IM were less disabled than those with IFN $\beta-1$ a SC or IFN $\beta-1 b$, and those on GA were older than those treated with IFN $\beta-1$ a SC or IM (see eTable 2). Patients were matched on their estimated probability of allocation to either compared medication (expressed as propensity score), with between 65 and $90 \%$ of the included patients retained in the subsequent head-to-head 
Table 1. Characteristics of the included patients.

\begin{tabular}{|c|c|c|c|c|}
\hline & IFN $\beta$-1a IM & IFN $\beta-1 \mathrm{a}$ SC & IFN $\beta-1 b$ & GA \\
\hline \multicolumn{5}{|l|}{ BASELINE } \\
\hline patients, number ( $\%$ females) & $832(71 \%)$ & $1379(70 \%)$ & $633(70 \%)$ & $482(73 \%)$ \\
\hline age at treatment start, years & $32.8 \pm 10 \cdot 0$ & $33.5 \pm 9.4$ & $34.6 \pm 9.4$ & $35.1 \pm 8.9$ \\
\hline disease duration, years & $2.9 \pm 2.6$ & $2.9 \pm 2.6$ & $2.9 \pm 2.6$ & $3.2 \pm 2.7$ \\
\hline baseline ARR & $1.6 \pm 1.1$ & $1.8 \pm 1.3$ & $1.7 \pm 1.1$ & $1.6 \pm 1.2$ \\
\hline \multicolumn{5}{|l|}{ disease course } \\
\hline CIS, number (\%) & $93(11 \%)$ & $59(4 \%)$ & $43(7 \%)$ & $23(5 \%)$ \\
\hline RRMS, number (\%) & $737(89 \%)$ & $1309(95 \%)$ & $581(92 \%)$ & $454(94 \%)$ \\
\hline RPMS, number (\%) & $2(0.2 \%)$ & $11(0.8 \%)$ & $9(1 \%)$ & $5(1 \%)$ \\
\hline disability, EDSS* & $2(1.0,2.5)$ & $2(1.5,3.0)$ & $2(1.0,3.0)$ & $2(1.0,2.5)$ \\
\hline EDSS $0-1.5$, number $(\%)$ & $407(49 \%)$ & $510(37 \%)$ & $248(39 \%)$ & $203(42 \%)$ \\
\hline EDSS $2-3.5$, number (\%) & $374(45 \%)$ & $705(51 \%)$ & $289(46 \%)$ & $237(49 \%)$ \\
\hline EDSS $4-8.5$, number $(\%)$ & $51(6 \%)$ & $164(12 \%)$ & $96(15 \%)$ & $42(9 \%)$ \\
\hline MS centres, number & 43 & 45 & 40 & 39 \\
\hline Time on treatment, months* & $41(24,69)$ & $47(28,77)$ & $45(24,84)$ & $44(25,74)$ \\
\hline \multicolumn{5}{|l|}{$\begin{array}{l}\text { MRI: hyperintense } \mathrm{T} 2 \text { lesions, } \\
\text { number }(\%)\end{array}$} \\
\hline availability & $491(59 \%)$ & $831(60 \%)$ & $312(49 \%)$ & $270(56 \%)$ \\
\hline 0 & $91(19 \%)$ & $141(17 \%)$ & $68(22 \%)$ & $75(28 \%)$ \\
\hline $1-8$ & $324(66 \%)$ & $572(69 \%)$ & $179(57 \%)$ & $145(54 \%)$ \\
\hline $9+$ & $76(15 \%)$ & $118(14 \%)$ & $65(21 \%)$ & $50(19 \%)$ \\
\hline \multicolumn{5}{|l|}{$\begin{array}{l}\text { MRI: contrast enhancing lesions, } \\
\text { number }(\%)\end{array}$} \\
\hline availability & $425(51 \%)$ & $713(52 \%)$ & $236(37 \%)$ & $202(58 \%)$ \\
\hline no & $324(76 \%)$ & $507(71 \%)$ & $191(81 \%)$ & $156(77 \%)$ \\
\hline yes & $101(24 \%)$ & $206(29 \%)$ & $45(19 \%)$ & $46(23 \%)$ \\
\hline \multicolumn{5}{|l|}{ Cerebrospinal fluid, number (\%) } \\
\hline availability & $332(40 \%)$ & $626(35 \%)$ & $180(28 \%)$ & $144(30 \%)$ \\
\hline abnormal & $304(92 \%)$ & $543(87 \%)$ & $162(90 \%)$ & $122(85 \%)$ \\
\hline normal & $28(8 \%)$ & $83(13 \%)$ & $18(10 \%)$ & $22(15 \%)$ \\
\hline \multicolumn{5}{|l|}{ STUDY END } \\
\hline $\begin{array}{l}\text { visit density (visits per year), } \\
\text { median (quartiles) }\end{array}$ & $2.1(1.1,4.0)$ & $1.9(1.0,3.7)$ & $1.7(0.9,3.5)$ & $1.9(1.0,3.6)$ \\
\hline \multicolumn{5}{|c|}{$\begin{array}{l}\text { further disease-modifying therapy, } \\
\text { number }(\%)\end{array}$} \\
\hline continued previous therapy $\dagger$ & $247(30 \%)$ & $550(40 \%)$ & $223(35 \%)$ & $207(43 \%)$ \\
\hline switched to IFN $\beta$-1a IM & 0 & $40(3 \%)$ & $36(6 \%)$ & $13(3 \%)$ \\
\hline switched to IFN $\beta$-1a SC & $189(23 \%)$ & 0 & $16(3 \%)$ & $34(7 \%)$ \\
\hline switched to IFN $\beta-1 b$ & $32(4 \%)$ & $8(0 \cdot 6 \%)$ & 0 & $14(3 \%)$ \\
\hline switched to GA & $48(6 \%)$ & $117(8 \%)$ & $38(6 \%)$ & 0 \\
\hline switched to fingolimod & $10(1 \%)$ & $22(2 \%)$ & $18(3 \%)$ & $14(3 \%)$ \\
\hline switched to teriflunomide & 0 & 0 & 0 & $1(0 \cdot 2 \%)$ \\
\hline switched to natalizumab & $24(3 \%)$ & $69(5 \%)$ & $29(5 \%)$ & $15(3 \%)$ \\
\hline switched to mitoxantrone & $1(0.1 \%)$ & $25(2 \%)$ & $10(2 \%)$ & $5(1 \%)$ \\
\hline enrolled in randomised trial & $2(0.2 \%)$ & 0 & $1(0.1 \%)$ & $2(0.4 \%)$ \\
\hline stopped previous therapy $\ddagger$ & $279(34 \%)$ & $548(40 \%)$ & $262(41 \%)$ & $177(38 \%)$ \\
\hline \multicolumn{5}{|c|}{$\begin{array}{l}\text { reason for treatment } \\
\text { discontinuation/switch, number }(\%)\end{array}$} \\
\hline data availability & $251(43 \%)$ & $416(50 \%)$ & $141(34 \%)$ & $94(34 \%)$ \\
\hline lack of tolerability & $40(16 \%)$ & $131(31 \%)$ & $60(43 \%)$ & $25(27 \%)$ \\
\hline
\end{tabular}


Table 1. (Continued)

\begin{tabular}{|c|c|c|c|c|}
\hline & IFN $\beta-1 \mathrm{a}$ IM & IFN $\beta$-1a SC & IFN $\beta-1 b$ & GA \\
\hline lack of effect & $138(55 \%)$ & $150(36 \%)$ & $34(24 \%)$ & $36(38 \%)$ \\
\hline $\begin{array}{l}\text { convenience / planned } \\
\text { discontinuation }\end{array}$ & $73(29 \%)$ & $135(32 \%)$ & $47(33 \%)$ & $33(35 \%)$ \\
\hline \multicolumn{5}{|c|}{$\begin{array}{l}\text { "median (interquartile range); otherwise mean } \pm \text { standard deviation are shown. } \\
\text { †on-study follow-up was terminated at the last recorded visit. } \\
\text { "discontinuation of previous therapy exceeding } 3 \text { months. } \\
\text { ARR: annualised relapse rate; CIS: clinically isolated syndrome; EDSS: Extended Disability Status Scale; GA: glatiramer acetate; } \\
\text { IFN: interferon; IM: intramuscular; MRI: magnetic resonance imaging; MS: multiple sclerosis; RPMS: relapsing-progressive mul- } \\
\text { tiple sclerosis; RRMS: relapsing-remitting multiple sclerosis; SC: subcutaneous. }\end{array}$} \\
\hline
\end{tabular}

comparisons. Average between-group differences in propensity scores were reduced by $96-97 \%$ (eFigure $1)$. The resulting six paired groups were closely matched on all recorded demographic and clinical parameters (Table 2). In addition, categorised MRI and cerebrospinal fluid variables (not included in the propensity calculations due to high proportion of missing data) did not markedly differ between the matched groups.

Average ARR recorded during the follow-up period ranged from 0.38 to 0.56 relapse/year (Table 3). Comparisons of ARR between the matched groups showed that patients treated with GA or IFN $\beta$-1a SC experienced fewer relapses than those receiving IFN $\beta$-1a IM or IFN $\beta-1 b \quad(p \leq 0.001)$. The observed mean differences were small (0.15-0.16 and 0.09-0.1 relapse/year for GA and IFN $\beta$-1a SC, respectively). Between $38 \%$ and $52 \%$ of all relapses were treated with steroids (steroid-treated ARR 0.17-0.25), with no statistically significant differences between the compared therapies and only with a nearly significant trend observed between IFN $\beta$ - 1 a SC and IFN $\beta$-1a IM (0.03 relapse/year, $p=0.07)$. The proportion of relapsefree patients (Figure 2) was significantly higher for GA in comparison with either IFN $\beta$-1a IM or IFN $\beta-1 b$ (hazard ratio $=1.36$ and 1.48 , respectively, $p \leq 0.02$ ). In addition, a marginally higher proportion of relapsefree patients was observed for IFN $\beta$-1a IM in comparison with IFN $\beta-1 \mathrm{~b}$ (hazard ratio $=1.28, p=0.05$ ). In contrast, we did not observe any differences in the proportions of patients free from 12-month sustained progression of disability (Figure 3, eFigure 2) in the analyses extending to 10 years.

The outcomes in the unmatched cohorts are shown in eTable 3 and eFigures 3-5. The sensitivity analyses within the groups matched in a 1:1 ratio retained $38 \%$ and $60 \%$ of patients from the primary analysis. With the exception of the proportion of relapse-free patients treated with IFN $\beta-1 \mathrm{a}$ IM vs. GA, the sensitivity analyses confirmed all observed effects (eTable 4). The Hodges-Lehmann sensitivity parameter $(\Gamma)$ showed that the ARR analyses were resistant to a hypothetical unknown confounder of a magnitude of $10-40 \%$ of the propensity score, with the exception of the comparison of IFN $\beta$-1a SC vs. IM, which was vulnerable to an unidentified confounder of any magnitude. The analysis of steroid-treated ARR was vulnerable to any unidentified confounders. The analysis of ARR contained $95 \%$ power to identify the minimum effect size of $0.07-0.15$ relapse/year. Finally, the sensitivity analysis of the proportions of patients free from 12-month sustained disability progression did not find any significant differences between the compared therapies (eTable 5).

\section{Discussion}

In this analysis of clinical practice data, we have directly compared effectiveness of the injectable immunomodulatory agents (IFN $\beta$ and GA preparations) in 3326 closely matched patients using pairwise comparisons with on-treatment follow-up spanning up to 10 years. In this cohort the patients treated with IFN $\beta$-1a SC or GA were at slightly but significantly lower risk of MS relapses than those treated with IFN $\beta$-1a IM or IFN $\beta-1 b$, as demonstrated by ARR and the proportions of relapse-free patients. We have found no difference in the rate of the first 12-month confirmed disability progression events between the compared medications.

Our study confirms and extends the outcomes of several previously published head-to-head RCTs. The EVIDENCE trial and its extension showed superior effect of IFN $\beta$-1a SC on relapse activity and active MRI lesions over a 2-year follow-up period compared with IFN $\beta-1$ a IM. ${ }^{2}$ Another RCT in 90 patients suggested superior effect of IFN $\beta$-1a SC on relapse activity compared with IFN $\beta$-1a IM. ${ }^{25}$ In the REGARD trial, no difference in the effect on relapse 


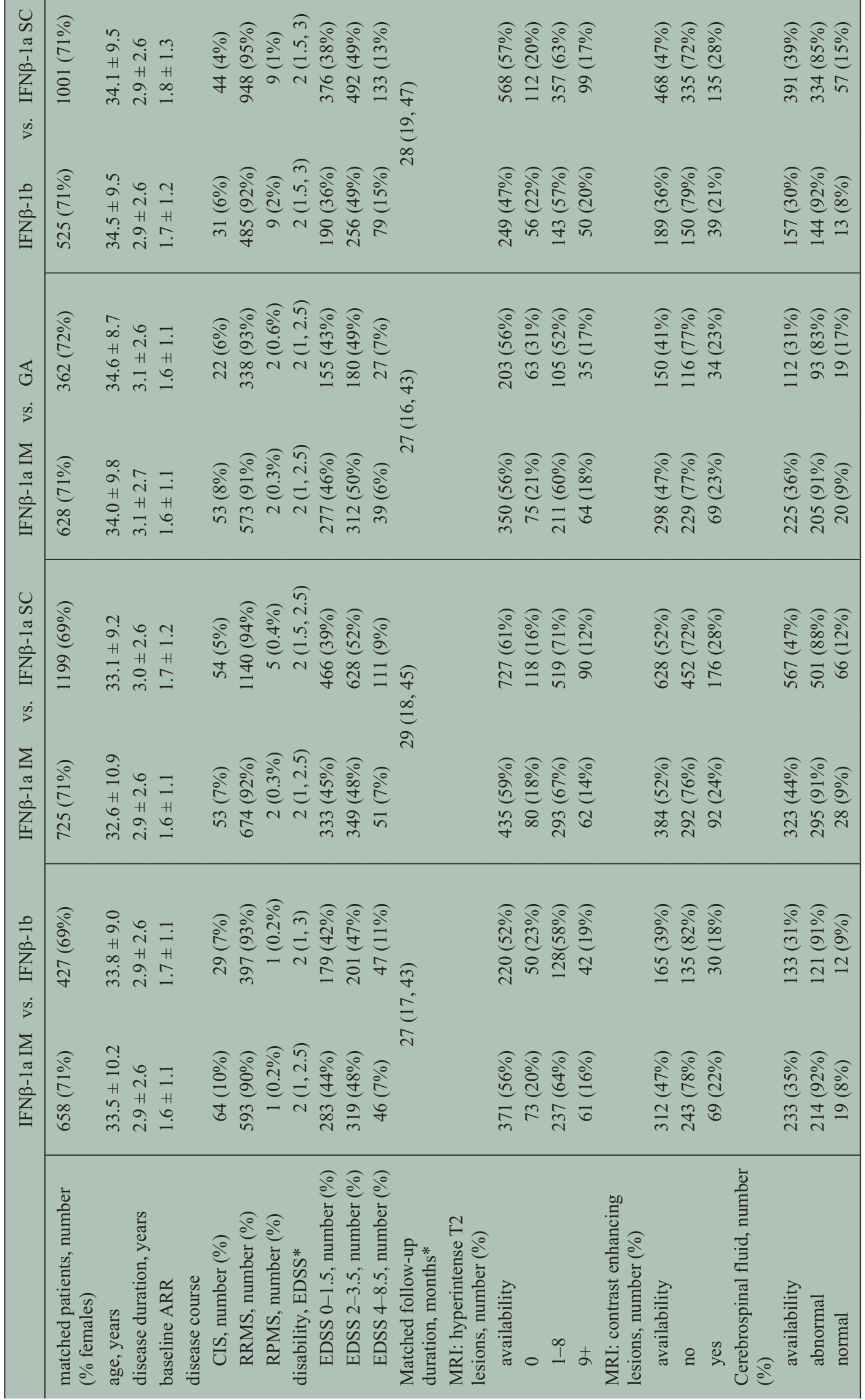




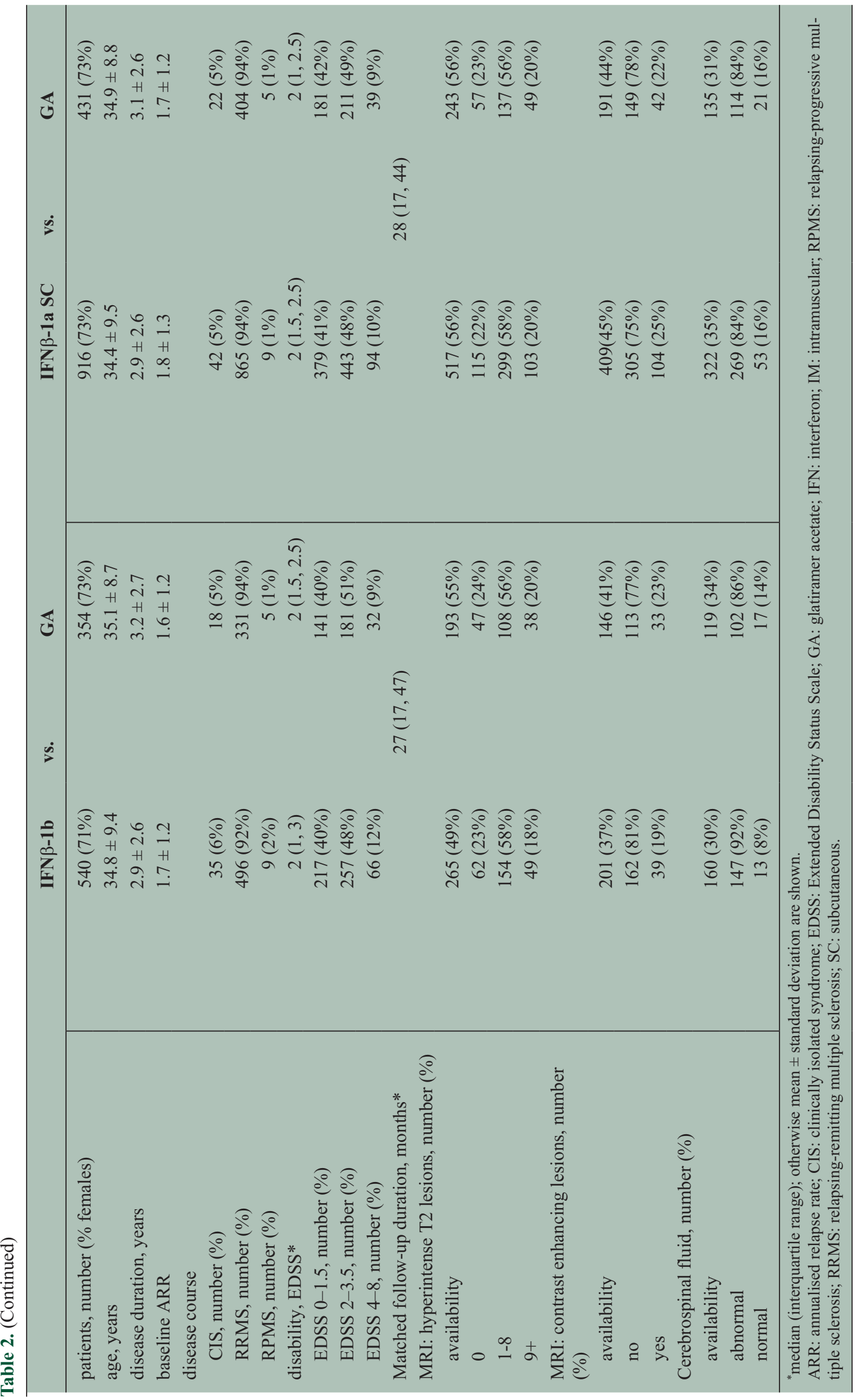


Table 3. Annualised relapse rates in matched cohorts.

\begin{tabular}{|c|c|c|c|c|c|c|}
\hline & \multicolumn{3}{|c|}{ all relapses } & \multicolumn{3}{|c|}{ relapses treated with steroids } \\
\hline & mean & SD & $p^{*}$ & mean & SD & $p^{*}$ \\
\hline Glatiramer acetate vs. & 0.38 & 0.63 & $10^{-5}$ & 0.17 & 0.37 & 0.1 \\
\hline IFN $\beta-1$ a IM & 0.53 & 0.81 & & 0.24 & 0.57 & \\
\hline Glatiramer acetate vs. & 0.40 & 0.66 & $10^{-4}$ & 0.21 & 0.45 & 0.4 \\
\hline IFN $\beta-1 b$ & 0.56 & 0.73 & & 0.23 & 0.49 & \\
\hline Glatiramer acetate vs. & 0.39 & 0.60 & 0.2 & 0.20 & 0.44 & 0.8 \\
\hline IFN $\beta-1$ a SC & 0.42 & 0.66 & & 0.19 & 0.42 & \\
\hline IFN $\beta-1$ a SC vs. & 0.44 & 0.58 & $10^{-4}$ & 0.21 & 0.40 & 0.07 \\
\hline IFN $\beta$-1a IM & 0.53 & 0.78 & & 0.24 & 0.52 & \\
\hline IFN $\beta-1$ a SC vs. & 0.45 & 0.66 & 0.001 & 0.18 & 0.43 & 0.7 \\
\hline IFN $\beta-1 b$ & 0.55 & 0.73 & & 0.21 & 0.48 & \\
\hline IFN $\beta$-1a IM vs. & 0.54 & 0.82 & 0.2 & 0.25 & 0.59 & 0.1 \\
\hline IFN $\beta-1 b$ & 0.53 & 0.71 & & 0.22 & 0.43 & \\
\hline
\end{tabular}

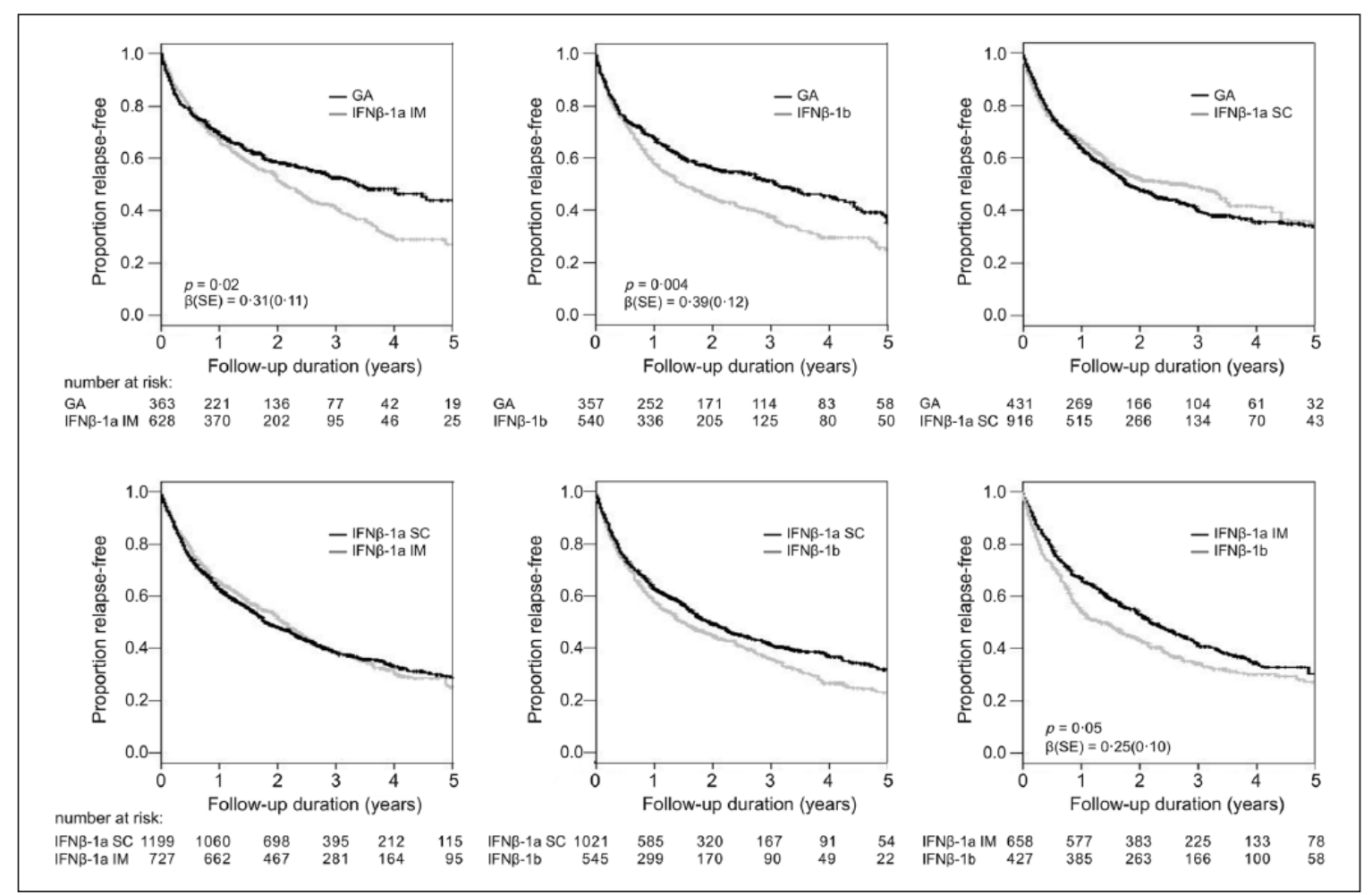

Figure 2. Proportions or patients free from relapses.

Proportions of relapse-free patients are shown for all pairwise comparisons of the studied therapies, within the propensity score-matched groups. Pairwise censoring was applied throughout. The comparisons were censored latest at 5 years from treatment onset; $p$-values indicate the outcomes of paired frailty models.

$\beta$ : coefficient; GA: glatiramer acetate; IFN: interferon; IM: intramuscular; SC: subcutaneous; SE: standard error of coefficient.

activity was observed between patients treated with IFN $\beta$-1a SC or GA. However, IFN $\beta$-1a SC showed a more pronounced reduction of active T2 lesions, while GA was associated with reduction in brain 


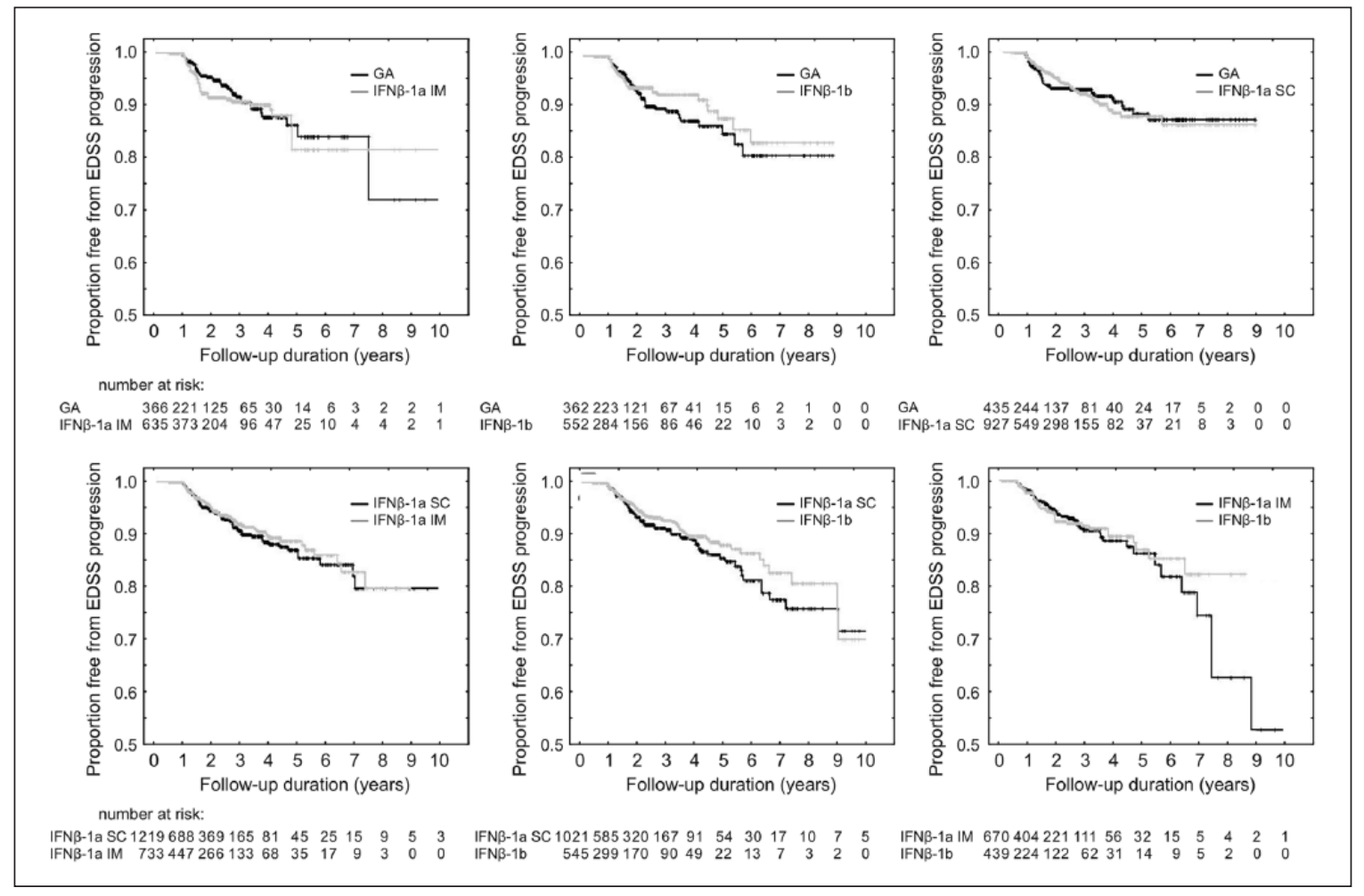

Figure 3. Proportions of patients with the first on-treatment disability progression event confirmed at 12 months. Disability progression events are shown for all pairwise comparisons of the studied therapies, within the propensity score-matched groups. Pairwise censoring was applied throughout. Frailty proportional hazards models did not identify any differences between any of the pairwise comparisons.

EDSS: Expanded Disability Status Scale; GA: glatiramer acetate; IFN: interferon; IM: intramuscular; SC: subcutaneous.

atrophy. ${ }^{5}$ The CombiRX trial showed that the effect of GA on relapse activity over 3 years was superior to that of IFN $\beta-1 \mathrm{a}$ IM. ${ }^{6}$ In addition, an RCT in 141 patients showed a more pronounced effect of IFN $\beta-1 \mathrm{a}$ $\mathrm{SC}$ on new cortical demyelinating lesions than GA or IFN $\beta-1$ a IM. ${ }^{26}$

In contrast, our study did not confirm the results of some other RCTs. The BEYOND trial did not find any differences in ARR between GA and IFN $\beta-1 b$ over 2 years, but showed superior effect of IFN $\beta-1 b$ on change in $\mathrm{T} 2$ lesion volume and number of new T2 lesions. ${ }^{1}$ The INCOMIN trial showed that the effect of IFN $\beta-1 \mathrm{~b}$ on relapse and MRI activity was superior to that of IFN $\beta$-1a IM. ${ }^{3}$ In addition, an unmatched retrospective cohort study in 546 patients, which was not adjusted for baseline differences between patients, reported comparable treatment outcomes among all four injectable immunomodulatory therapies. ${ }^{27} \mathrm{It}$ is of interest that while several of these studies showed an association between interferon dosage and its therapeutic efficacy, our present analysis showed comparable effectiveness of low-dose IFN $\beta$-1a IM and IFN $\beta-1 b$. A possible explanation may involve the role of neutralising antibodies, whereby IFN $\beta-1 \mathrm{a}$ IM administered once weekly is known to be less immunogenic than IFN $\beta$ - $1 \mathrm{a}$ and IFN $\beta-1 \mathrm{~b}$ administered subcutaneously multiple times per week. ${ }^{28}$ However, it should be noted that our previous analysis comparing two doses of IFN $\beta$-1a SC did not identify any dosedependent effects. ${ }^{22}$ It should also be noted that our analysis did not show any statistically significant differences in the frequency of steroid-treated relapses. Besides the lack of power determined by the relatively low steroid ARR, it could be speculated that this may reflect difference in the on-treatment relapse severity, of which steroid therapy may be indicative.

A recent Cochrane review including five trials comparing IFN $\beta$ and GA reported no differences in relapse and disability outcomes between the compared preparations at 2 years and a slightly lower ARR at 3 years in GA relative to IFN $\beta$-1a IM (as shown in the CombiRX trial). ${ }^{29}$ This suggests that any differences in relapse activity between the injectable immunomodulators are minimal and detectable only on sufficient follow-up duration. In their network meta-analysis, Filippini and colleagues used a combination of direct and indirect 
comparisons to estimate relative treatment efficacy among the commonly used disease-modifying therapies, including IFN $\beta$ and GA. ${ }^{30}$ In agreement with our findings, the meta-analysis showed superiority of IFN $\beta$ - 1 a SC over the other IFN $\beta$ preparations in their effect on relapse outcomes. In addition, unlike our analysis, it reported superiority of IFN $\beta$-1a SC over GA. Finally, the meta-analysis did not find any statistically significant differences in the disability endpoints, thus converging with our conclusions. The authors attributed this to the limited follow-up in the available RCTs (2-3 years). It is worth noting that in a number of instances, including the comparisons between GA vs. IFN $\beta$-1a SC or IFN $\beta-1 b$, the quality of the evidence was deemed insufficient for a reliable comparison of relapse outcomes. Another systematic review, which analysed outcomes of the RCTs completed before 2010, reported a superior effect of IFN $\beta-1 b$ on relapse activity when compared with IFN $\beta-1$ a SC. ${ }^{31}$ Similarly to the metaanalysis, the review did not find any differences in the disability outcomes. Our study has confirmed that the injectable immunomodulatory therapies do not differ in their effects on accumulation of disability over extended follow-up in routine clinical practice, despite small differences in the effect on relapses.

Network meta-analysis assumes consistency between the compared trials, evaluated using visual inspection of forest plots or by $I^{2}$ statistic, whose power to detect important clinical and methodological effect modifiers is low. ${ }^{30}$ Therefore, the outcomes of network meta-analyses may be susceptible to confounding introduced by the heterogeneity of indirectly compared trial populations. Our analysis used matched head-to-head comparisons with uniform inclusion criteria and follow-up protocol, and was therefore relatively resistant to population heterogeneity. To adjust for potential confounders of treatment indication, we used a previously validated propensity score-based procedure. ${ }^{22}$ We used variable matching ratio, which is more efficient in eliminating indication bias than fixed ratio and leads to only a minimal increase in variance. ${ }^{32}$ To eliminate any attrition bias (which favours therapy perceived as less effective), we have applied pairwise censoring, where any discontinuation of therapy or follow-up triggered censoring of both matched patients. ARR observed in this study was compatible with ARR reported in other contemporary studies. ${ }^{33}$ In comparison with the previous RCTs, our study had greater power to evaluate disability outcomes, mainly due to the longer clinical follow-up (median 3.7 years).

The analysed data were collected as part of routine practice and therefore could be subject to selection, performance, or detection bias. The high similarity of the follow-up density between the treatment cohorts does not suggest presence of selection bias due to preferential inclusion of any therapy. Given that the present analysis lacked comparison against an untreated population (as we were unable to identify an unbiased natural-history cohort in the MSBase registry), the study was not affected by an immortal-time bias. Detection bias could have been introduced by the varying treatment preferences and follow-up protocols at the multiple contributing centres. MSBase is a prospective cohort study largely conducted at large university MS centres with certifications to conduct RCTs. We have applied the minimum data quality definitions to ameliorate detection bias but we acknowledge that we were unable to control the input quality at the level of a prospectively designed RCT. It should be noted that neither the previously conducted head-to-head trials were free from these biases, as they were conducted as open-label or single-blinded studies. On the other hand, our present analysis was free from attrition, reporting, or funding bias. Finally, it should be noted that the information about treatment safety for the analysed preparations was relatively incomplete and therefore not reported.

Our observations in a large MS clinical practice cohort suggest that subcutaneous IFN $\beta$ - $1 \mathrm{a}$ and GA are associated with a slightly superior effect relative to intramuscular IFN $\beta-1 \mathrm{a}$ and IFN $\beta$ - $1 \mathrm{~b}$ on reducing frequency of relapses but not on delaying accumulation of irreversible neurological disability. Comparisons with newer therapies in the clinical practice setting are eagerly awaited.

\section{Acknowledgements}

MSBase study group co-investigators and contributors:

From Hopital Tenon, Paris, France, Dr Etienne Roullet; from Jahn Ferenc Teaching Hospital, Budapest, Hungary, Dr Csilla Rozsa and Dr Krisztian Kasa; from Central Clinical Emergency Military Hospital, Bucharest, Romania, Dr Carmen-Adella Sirbu; from the Geelong Hospital, Geelong, Australia, Dr Cameron Shaw; from the Westmead Hospital, Sydney, Australia, Dr Steve Vucic; from the Clinic of Neurology Clinical Center, Skopje, Macedonia, Dr Tatjana Petkovska-Boskova; from the New York University Langone Medical Center, New York, USA, Dr Joseph Herbert and Dr Ilya Kister; from the Bombay Hospital Institute of Medical Sciences, Mumbai, India, Dr Bhim Singhal; from the Amiri Hospital, Kuwait City, Kuwait, Dr Raed Alroughani; from the Instituto de Neurociencias, Cordoba, Argentina, Dr Elizabeth Alejandra Bacile Bacile; from the Hospital Ecoville, Brazil, Dr Walter 
Oleschko Arruda; from the Centre hospitalier del'Universite de Montreal, Hopital Notre-Dame, Canada, Dr Elaine Roger and Dr Pierre Despault; from the Royal Melbourne Hospital, Australia, Dr Mark Marriott, Dr Anneke Van der Walt, Dr John King, Dr Jill Byron, Ms Lisa Morgan and Ms Eloise Hinson; from Box Hill Hospital, Monash University, Australia, Ms Jodi Haartsen; from Rodanotech, Switzerland, Mr Samir Mechati, Mr Erich Bianchi, Mr Alexandre Bulla and Mr Matthieu Corageoud; from Department of Neuroscience, Imaging and Clinical Sciences, University 'G. d'Annunzio', Italy, Dr Giovanna De Luca, Dr Valeria Di Tommaso, Dr Daniela Travaglini, Dr Erika Pietrolongo, Dr Maria di Ioia, Dr Deborah Farina and Dr Luca Mancinelli; from Hospital Italiano, Argentina, Dr Juan Ignacio Rojas and Dr Liliana Patrucco; from Ospedale di Macerata, Italy, Dr Elisabetta.

\section{Conflict of interests}

Alessandra Lugaresi is a Bayer Schering, Biogen Idec, Genzyme, Merck Serono Advisory Board Member. She received travel grants and honoraria from Bayer Schering, Biogen Idec, Merck Serono, Novartis, Sanofi and Teva, research grants from Bayer Schering, Biogen Idec, Merck Serono, Novartis, Sanofi and Teva, travel and research grants from the Associazione Italiana Sclerosi Multipla.

Cavit Boz did not declare any competing interests.

Celia Oreja-Guevara received honoraria as consultant on scientific advisory boards from Biogen Idec, Bayer Schering, Merck Serono, Teva and Novartis; has participated in clinical trials/other research projects by Biogen Idec, GSK, Teva and Novartis.

Dana Horakova received speaker honoraria and consulting fees from Biogen Idec, Merck Serono, Teva and Novartis, as well as support for research activities from Biogen Idec.

Daniele Spitaleri received honoraria as a consultant on scientific advisory boards by Bayer Schering, Novartis and Sanofi-Aventis and compensation for travel from Novartis, Biogen Idec, Sanofi Aventis, Teva and Merck-Serono.

Edgardo Cristiano received honoraria as consultant on scientific advisory boards by Biogen Idec, Bayer Schering, Merck-Serono, Genzyme and Novartis; has participated in clinical trials/other research projects by Merck-Serono, Roche and Novartis.

Eugenio Pucci served on scientific advisory boards for Genzyme and Biogen Idec; he has received honoraria and travel grants from Sanofi Aventis, UCB, Lundbeck, Novartis, Bayer Schering, Biogen Idec,
Merck Serono, Genzyme and Teva; he has received travel grants from Associazione Marchigiana Sclerosi Multipla e altre malattie neurologiche.

Eva Havrdova received speaker honoraria and consultant fees from Biogen Idec, Merck Serono, Novartis, Genzyme and Teva, as well as support for research activities from Biogen Idec and Merck Serono.

Francois Grand'Maison received honoraria from Biogen Idec, Genzyme, Novartis and Roche.

Fraser Moore has participated in clinical trials sponsored by EMD Serono and Novartis.

Freek Verheul did not declare any competing interests.

Gerardo Iuliano had travel/accommodations/meeting expenses funded by Bayer Schering, Biogen Idec, Merck Serono, Novartis, Sanofi Aventis, and Teva

Guillermo Izquierdo received speaking honoraria from Biogen Idec, Novartis, Sanofi, Serono and Teva.

Helmut Butzkueven has served on scientific advisory boards for Biogen Idec, Novartis and SanofiAventis and has received conference travel support from Novartis, Biogen Idec and Sanofi Aventis. He serves on steering committees for trials conducted by Biogen Idec and Novartis, and has received research support from Merck Serono, Novartis and Biogen Idec.

Jeannette Lechner-Scott has accepted travel compensation from Novartis, Biogen and Merck Serono. Her institution receives the honoraria for talks and advisory board commitment and also clinic support from Bayer Health Care, Biogen Idec, CSL, Genzyme Sanofi, Merck Serono and Novartis.

Jose Antonio Cabrera-Gomez did not declare any competing interests.

Marc Girard received consulting fees from Teva Canada Innovation, Biogen Idec, Novartis and Genzyme Sanofi; lecture payments from Teva Canada Innovation, Novartis and EMD Serono and a research grant from Canadian Institutes of Health Research.

Maria Laura Saladino did not declare any competing interests.

Maria Pia Amato received honoraria as consultant on scientific advisory boards by Biogen Idec, Bayer Schering, Merck-Serono, Teva and Sanofi-Aventis; has received research grants by Biogen Idec, Bayer Schering, Merck-Serono, Teva and Novartis.

Maria Trojano received speaking honoraria from Biogen Idec, Bayer Schering, Sanofi Aventis, Merck-Serono, Teva and Novartis; has received research grants from Biogen Idec, Merck-Serono, and Novartis. 
Mark Paine received travel grants from Novartis, Bayer Schering, Merck-Serono, Biogen Idec and honoraria from Novartis, BioCSL, Bayer Schering, and Biogen Idec.

Mark Slee has participated in, but not received honoraria for, advisory board activity for Biogen Idec, Merck Serono, Bayer Schering, Sanofi Aventis and Novartis.

Michael Barnett has served on scientific advisory boards for Biogen Idec, Novartis and Genzyme and has received conference travel support from Biogen Idec and Novartis. He serves on steering committees for trials conducted by Novartis. His institution has received research support from Biogen Idec, MerckSerono and Biogen Idec.

Orla Gray received honoraria as consultant on scientific advisory boards for Biogen Idec, Merck Serono and Novartis; has received travel grants from Biogen Idec, Merck Serono and Novartis; has participated in clinical trials by Biogen Idec and Merck Serono.

Pierre Duquette did not declare any competing interests.

Pierre Grammond is a Novartis, Teva-neuroscience, Biogen Idec advisory board member, consultant for Merck Serono, received payments for lectures by Merck Serono, Teva-Neuroscience and Canadian Multiple sclerosis society, and received grants for travel from Teva-Neuroscience and Novartis.

Raymond Hupperts received honoraria as consultant on scientific advisory boards from Merck-Serono, Biogen Idec, Genzyme-Sanofi and Teva, research funding from Merck-Serono and Biogen Idec, and speaker honoraria from Sanofi-Genzyme.

Ricardo Fernandez-Bolanos did not declare any competing interests.

Roberto Bergamaschi received speaker honoraria from Bayer Schering, Biogen Idec, Genzyme, Merck Serono, Novartis, Sanofi-Aventis, Teva; research grants from Bayer Schering, Biogen Idec, Merck Serono, Novartis, Sanofi-Aventis, Teva; congress and travel/accommodation expense compensations by Almirall, Bayer Schering, Biogen Idec, Genzyme, Merck Serono, Novartis, SanofiAventis, Teva.

Shlomo Flechter did not declare any competing interests.

Tomas Kalincik received compensation for travel and consultancy or speaker honoraria from Novartis, Biogen Idec, Genzyme, Sanofi Aventis, Teva, BioCSL and Merck Serono.

Vilija Jokubaitis has received compensation for travel from Novartis.
Vincent Van Pesch has served on advisory boards for Biogen Idec and Genzyme; has received travel grants from Biogen Idec, Bayer Schering, Sanofi Aventis, Merck Serono and Novartis Pharma; has received consultancy fees from Biogen Idec, Teva and Novartis Pharma; has received research grants from Bayer Schering.

\section{Funding}

The work was supported by the Multiple Sclerosis Research Australia Postdoctoral Fellowship [11-054] and NHMRC Early Career Award (Clinical) to TK [1071124], NHMRC Career Development Award (Clinical) to HB [628856], NHMRC Project Grant [1032484], NHMRC Centre for Research Excellence [1001216] and the MSBase Foundation. The MSBase Foundation is a not-for-profit organization that receives support from Merck Serono, Biogen Idec, Novartis Pharma, Bayer Schering, Sanofi-Aventis and BioCSL. The study was conducted separately and apart from the guidance of the sponsors. TK had full access to all the data and takes responsibility for integrity of the data and accuracy of the data analysis.

\section{References}

1. O'Connor P, Filippi M, Arnason B, et al. 250 microg or 500 microg interferon beta-1b versus $20 \mathrm{mg}$ glatiramer acetate in relapsing-remitting multiple sclerosis: A prospective, randomised, multicentre study. Lancet Neurol 2009; 8: 889-897.

2. Schwid S and Panitch H. Full results of the Evidence of Interferon Dose-Response-European North American Comparative Efficacy (EVIDENCE) study: A multicenter, randomized, assessor-blinded comparison of low-dose weekly versus high-dose, high-frequency interferon beta-1a for relapsing multiple sclerosis. Clin Ther 2007; 29: 2031-2048.

3. Durelli L, Verdun E, Barbero P, et al. Everyother-day interferon beta- $1 \mathrm{~b}$ versus once-weekly interferon beta-1a for multiple sclerosis: Results of a 2-year prospective randomised multicentre study (INCOMIN). Lancet 2002; 359: 1453-1460.

4. Koch Henriksen N, Sorensen PS, Christensen T, et al. A randomized study of two interferon-beta treatments in relapsing-remitting multiple sclerosis. Neurology 2006; 66: 1056-1060

5. Mikol D, Barkhof F, Chang P, et al. Comparison of subcutaneous interferon beta-1a with glatiramer acetate in patients with relapsing multiple sclerosis (the REbif vs Glatiramer Acetate in Relapsing MS Disease [REGARD] study): A multicentre, randomised, parallel, open-label trial. Lancet Neurol 2008; 7: 903-914. 
6. Lublin FD, Cofield SS, Cutter GR, et al. Randomized study combining interferon and glatiramer acetate in multiple sclerosis. Ann Neurol 2013; 73: 327-340.

7. Trojano M, Pellegrini F, Paolicelli D, et al. Observational studies: Propensity score analysis of non-randomized data. Int MS J 2009; 16: 90-97.

8. Rosenbaum PR and Rubin DB. Reducing bias in observational studies using subclassification on the propensity score. J Am Stat Assoc 1984; 79: 516-524.

9. Trojano M, Pellegrini F, Fuiani A, et al. New natural history of interferon-beta-treated relapsing multiple sclerosis. Ann Neurol 2007; 61: 300-306.

10. Conway DS, Miller DM, O'Brien RG, et al. Long term benefit of multiple sclerosis treatment: An investigation using a novel data collection technique. Mult Scler 2012; 18: 1617-1624.

11. Shirani A, Zhao Y, Karim ME, et al. Association between use of interferon beta and progression of disability in patients with relapsing-remitting multiple sclerosis. JAMA 2012; 308: 247-256.

12. Kalincik T, Vivek V, Jokubaitis V, et al. Sex as a determinant of relapse incidence and progressive course of multiple sclerosis. Brain 2013; 136 : 3609-3617.

13. PRISMS (Prevention of Relapses and Disability by Interferon beta-1a Subcutaneously in Multiple Sclerosis) Study Group. Randomised double-blind placebo-controlled study of interferon beta-1a in relapsing/remitting multiple sclerosis. Lancet 1998; 352: 1498-1504.

14. Butzkueven H, Chapman J, Cristiano E, et al. MSBase: An international, online registry and platform for collaborative outcomes research in multiple sclerosis. Mult Scler 2006; 12: 769-774.

15. Polman CH, Reingold SC, Edan G, et al. Diagnostic criteria for multiple sclerosis: 2005 revisions to the "McDonald Criteria". Ann Neurol 2005; 58: 840-846.

16. Polman $\mathrm{CH}$, Reingold SC, Banwell B, et al. Diagnostic criteria for multiple sclerosis: 2010 revisions to the McDonald criteria. Ann Neurol 2011; 69: 292-302.

17. Lublin FD and Reingold SC. Defining the clinical course of multiple sclerosis: Results of an international survey. National Multiple Sclerosis Society (USA) Advisory Committee on Clinical Trials of New Agents in Multiple Sclerosis. Neurology 1996; 46: 907-911.

18. Schumacher GA, Beebe G, Kibler RF, et al. Problems of experimental trials of therapy in multiple sclerosis: Report by the Panel on the Evaluation of Experimental Trials of Therapy in Multiple Sclerosis. Ann N Y Acad Sci 1965; 122: 552-568.

19. R Development Core Team. $R$ : A language and environment for statistical computing. Vienna,
Austria: R Foundation for Statistical Computing, 2011.

20. Ho DE, Imai K, King G, et al. Matching as nonparametric preprocessing for reducing model dependence in parametric causal inference. Polit Anal 2006; 15: 199-236.

21. Imai K, King G and Lau O. Toward a common framework for statistical analysis and development. $J$ Comput Graph Stat 2008; 17: 892-913.

22. Kalincik T, Spelman T, Trojano M, et al. Persistence on therapy and propensity matched outcome comparison of two subcutaneous interferon beta 1a dosages for multiple sclerosis. PLoS One 2013; 8: e63480.

23. Rosenbaum PR. Observational studies. 2nd ed. New York, NY: Springer-Verlag, 2002.

24. Sekhon J. Multivariate and propensity score matching software with automated balance optimization: The matching package for R. J Stat Software 2011; 42.

25. Etemadifar M, Janghorbani M and Shaygannejad V. Comparison of Betaferon, Avonex, and Rebif in treatment of relapsing-remitting multiple sclerosis. Acta Neurol Scand 2006; 113: 283-287.

26. Calabrese M, Bernardi V, Atzori M, et al. Effect of disease-modifying drugs on cortical lesions and atrophy in relapsing-remitting multiple sclerosis. Mult Scler 2012; 18: 418-424.

27. Gobbi C, Zecca C, Linnebank M, et al. Swiss analysis of multiple sclerosis: A multicenter, noninterventional, retrospective cohort study of diseasemodifying therapies. Eur Neurol 2013; 70: 35-41.

28. Goodin DS, Frohman EM, Hurwitz B, et al. Neutralizing antibodies to interferon beta: Assessment of their clinical and radiographic impact: an evidence report: Report of the Therapeutics and Technology Assessment Subcommittee of the American Academy of Neurology. Neurology 2007; 68: 977-984.

29. La Mantia L, Di Pietrantonj C, Rovaris M, et al. Interferons-beta versus glatiramer acetate for relapsing-remitting multiple sclerosis. Cochrane Database Syst Rev 2014; 7: CD009333.

30. Filippini G, Del Giovane C, Vacchi L, et al. Immunomodulators and immunosuppressants for multiple sclerosis: A network meta-analysis. Cochrane Database Syst Rev 2013; 6: CD008933-CD.

31. Smith B, Carson S, Fu R, et al. Drug class review: Disease-modifying drugs for multiple sclerosis: final update 1 report [Internet]. (2010, accessed 12 Oct 2013).

32. Ming K and Rosenbaum PR. Substantial gains in bias reduction from matching with a variable number of controls. Biometrics 2000; 56: 118-124.

33. Khan O, Rieckmann P, Boyko A, et al. Three times weekly glatiramer acetate in relapsing-remitting multiple sclerosis. Ann Neurol 2013; 73: 705-713.
Visit SAGE journals online http://msj.sagepub.com

(SAGE journals 